\title{
CHROMATOGRAPHIC DETERMINATION OF SOME CORTICOSTEROIDS, WITH SPECIAL REFERENCE TO HORSE DOPING
}

\author{
B. SCHUBERT \\ Government Laboratory for Forensic Chemistry, Department of Doping Analyses, \\ 10401 Stockholm 60, Sweden
}

\begin{abstract}
Some chromatographic procedures which can be used to detect and determine certain corticosteroids in samples from racehorses, are described. These procedures include thin-layer gas and high pressure liquid chromatography.
\end{abstract}

\section{THE DETECTION OF DOPING AGENTS IN BLOOD}

\author{
M. DONIKE, Priv.Doz.Dr.
}

An der Bottmühle 2, 5000 Köln 1, Germany \begin{abstract}
laboratory.
\section{Screening Procedure for Doping Agents in Urine}

The basis of our techniques for the detection of doping agents in urine is the screening procedures for volatile and slightly volatile drugs, devised for the $X X$ Olympic Games, Munich, 1972. The procedure covers most of the common phenylethylamines and some neutral drugs like nikethamide, leptazol and caffeine.
\end{abstract}

ABSTRACT

Gas chromatographic screening procedures have been evaluated which permit the detection of stimulants and sedatives in blood after administration of pharmacological doses. The techniques actually used in sample preparations and gas chromatographic work are presented as well as examples of pharmacokinetic studies and positive dope cases. The use of sensitive and selective detectors like the nitrogen-specific detector or a mass spectrometer is absolutely essential for routine work, as for non-specific detectors the number of "false positives" leads to an intolerable work load for the

1. Only one partition step: $5 \mathrm{ml}$ of urine are extracted with $2 \mathrm{ml}$ of ether after addition of $0.5 \mathrm{ml} 5 \mathrm{~N} \mathrm{KOH}, 3.5 \mathrm{~g} \mathrm{Na}_{2} \mathrm{SO}_{4}$ and 25 $\mu \mathrm{g}$ of diphenylamine as internal standard. The ether phase is used for gas-chromatographic analysis without concentration.

2. Chromatography is achieved on Apiezon L on Chromosorb W, washed with $\mathrm{KOH}$ and coated with $2 \%$ Igepal $\mathrm{CO} 880$.

3. A nitrogen specific detector (N-FID, Hewlett-Packard) is used because of its high sensitivity and selectivity.

4. A temperature programme with a gradient of $20^{\circ} \mathrm{C} /$ minute is used to shorten analysis time. The analysis cycle - injection, GC-analysis, cooling, equilibration and injection - lasts only 15 minutes.
5. Automatic injection guarantees a high reproducibility of retention times, which is a prerequisite of automatic evalution of the analytical data by computer.

Fig. 1 (a-d) demonstrates the potential of this screening procedure.

\section{Screening Procedure for Doping Agents in Blood}

Three reasons exist for attempting to detect doping agents in blood, which are valid for both human and veterinary sport:

1. The direct relationship between blood concentration and pharmacological activity.

2. The impossibility of obtaining urine in some cases.

3. The improved analytical methods of today which make it possible to detect blood levels of doping agents.

The change from urine to blood as a dope detection fluid presented many problems. Finally, we succeeded in screening blood for many doping agents. The character- 